\title{
Role of Purinome, A Complex Signaling System, In Glioblastoma Aggressiveness
}

\author{
Patricia Giuliani ${ }^{1,2 \dagger}$, Marzia Carluccio ${ }^{1,2 \dagger}$ and Renata Ciccarelli ${ }^{1,2 \dagger *}$ \\ ${ }^{1}$ Department of Medical, Oral and Biotechnological Sciences, 'G. D'Annunzio' University of Chieti-Pescara, Chieti, Italy, ${ }^{2}$ Center \\ for Advanced Studies and Technology (CAST), 'G. D'Annunzio' University of Chieti-Pescara, Chieti, Italy
}

Keywords: glioblastoma multiforme (GBM), purinome, adenine-based compounds, purine metabolizing enzymes, purinergic receptors

\section{INTRODUCTION}

Many recent papers dealing with glioblastoma multiforme (GBM), the most common/lethal human brain tumor, are aimed at identifying new druggable targets, hopefully useful for more effective therapies than the current ones. In this context, the purinergic system, present in all living cells, is arousing considerable interest, mainly in relation to adenine-based compounds. It is composed of:

OPEN ACCESS

Edited by: Henning Ulrich, University of São Paulo, Brazil

Reviewed by: Claudiana Lameu, University of São Paulo, Brazil

*Correspondence: Renata Ciccarell renata.ciccarelli@unich.it

${ }^{\dagger}$ These authors have contributed equally to this work

Specialty section:

This article was submitted to Experimental Pharmacology and

Drug Discovery,

a section of the journal

Frontiers in Pharmacology

Received: 23 November 2020 Accepted: 07 January 2021

Published: 05 February 2021

Citation:

Giuliani $P$, Carluccio $M$ and Ciccarelli R (2021) Role of Purinome, A Complex Signaling System, In Glioblastoma Aggressiveness. Front. Pharmacol. 12:632622. doi: 10.3389/fphar.2021.632622
(1) intracellular purine nucleotides, nucleosides and nucleobases involved in fundamental biological processes such as cell duplication, chemical energy supply, intracellular signaling, and protein metabolism regulation (Frenguelli and Dale, 2020);

(2) extracellular purines (the same found intracellularly), among which adenine-based nucleotides/ nucleosides behave as signal molecules interacting with specific receptors, namely P1 and P2 for adenosine (ADO) and ATP/ADP, respectively (Burnstock, 2018);

(3) a wide array of intra/extracellular metabolizing enzymes (Yegutkin, 2014);

(4) transporters, mostly deputed to regain nucleosides/bases to restore the intracellular purine pool (Choi and Berdis, 2012).

The advent of the "omic" era has induced scientists to indicate this complex network as "purinome", to emphasize that its components act in close/interactive fashion. This concerted activity maintains cells in homeostatic/physiological conditions, while purinome imbalance causes/is implicated in profound alterations of normal cell biological activities, thus contributing to malignant cell transformation toward more aggressive phenotypes, as shown in different tumors (Boison and Yegutkin, 2019; Campos-Contreras et al., 2020), including GBM (Giuliani et al., 2018; Zhou et al., 2020). Here, we exemplified some emerging alterations of purinome, highlighting how they influence GBM progression, in order to outline a picture, which can be the natural premise for suggesting new checkpoints of GBM aggressiveness.

\section{ROLE OF ADENOSINE TRIPHOSPHATE METABOLIZING ECTO-ENZYMES AND SIGNALS IN GLIOBLASTOMA AGGRESSIVENESS}

Notoriously, nucleotides, once released from cells, act on different $\mathrm{P} 2$ receptors divided into two subfamilies including eight metabotropic P2Y and seven ionotopic P2X receptors (Burnstock, 2018). Nucleotide receptor activity is finely tuned by ectoenzymes (Zimmermann et al., 2012). Of these, ectonucleoside triphosphate diphosphohydrolase (E-NTPDases) belong to the CD39 family that 



FIGURE 1 | Adenine-based purines are ubiquitous compounds present at intra- and intracellular levels in normal (i.e., astrocytes) and tumoral (i.e., GSCs) cells. Left panel: ATP is released by virtually all cell types, and is usually metabolized by ecto-enzymes into adenosine (ADO) that is regained by cells through selective transporters (carriers). Right panel: In tumors, including GBM, extracellular purine amount is increased in pericellular tumor fluid by cell membrane leakage and also by low metabolism of ATP by ecto-nucleotidases coupled to increased activity of the ectoenzyme (CD73) converting AMP into ADO. The interaction of extracellular ATP or ADO with specific receptors, the expression of which has been found increased in GSCs such as ADO/A3 and/or ATP/P2X7 receptors, activates processes contributing to transform tumor cells in phenotypes more aggressive and resistant to chemotherapy. Additionally, GBMs frequently show homozygous deletion of methylthioadenosine phosphorylase (MTAP), an enzyme working in the purine/methionine salvage pathway to convert methylthioadenosine (MTA) formed during polyamine biosynthesis into adenine and methionine. MTAP loss favors GSC formation with increased aggressiveness (Hansen et al., 2019) and is associated with poor clinical outcome of GBM patients (Zeppernick et al., 2008). In both panels, changes in the receptors expression or purine levels are highlighted by increasing or decreasing the character size and/ or by using bold characters. AK, adenosine kinase; NTs, nucleotidases; SAH, S-adenosyl hydroxylase, converting MTA into adenosine.

comprises eight subtypes, of which NTPDases1, 2 and 3 are present in the brain. Moreover, three subtypes of ectonucleoside pyrophosphatase/phosphodiesterases (E-NPPases) have been identified, being NPP2 and 3 expressed in brain glial cells (Braganhol et al., 2020), whereas tissue-nonspecific alkaline phosphatase (NTAP) is abundant in brain and prevailingly involved in the regulation of neuronal activity (Fonta et al., 2004). Finally, ecto-5'-nucleotidase/CD73 (ecto-5'-NT/CD73) is also a selective mesenchymal marker that, in tandem with CD39, generates ADO, thus contributing to the immunosuppressive potential of mesenchymal stromal cells (Kakiuchi et al., 2020).

Alterations in these extracellular/membrane purinome components are evident in GBM. While in normal cerebral tissue extracellular ATP levels are determined by nucleotide release from healthy neural cells (Burnstock, 2020), in pathological conditions including tumors, ATP levels are increased also through membrane leakage of damaged/dying cells, mainly in the hypoxic tumor core (Di Virgilio et al., 2018). However, differently from normal cultured astrocytes (Morrone et al., 2006), a low extracellular ATP metabolism was found in glioma cells (Wink et al., 2003). Further findings confirmed that such metabolic reduction is involved in GBM growth and progression (Braganhol et al., 2020) (Figure 1). Again, GBM cell lines and stem-like cells (GSCs, which support tumor recurrence) (Taga and Tabu, 2020), are sensitive to cytotoxicity of high ATP concentrations, while lower nucleotide levels (below $1 \mathrm{mM}$ ) would favor tumor growth (Morrone et al., 2005). Accordingly, co-injection of apyrase, an ATP/ADP scavenger, in C6 rat glioma experimental model, reduced tumor size when compared with that from rats untreated or exposed to inactivated apyrase (Morrone et al., 2006).

In parallel, other findings demonstrated that ATP receptors, i.e., the subtypes P2Y1, P2Y2, P2Y12 and P2X7, are important in GBM pathology (Giuliani et al., 2018; Braganhol et al., 2020). Among them, the $\mathrm{P} 2 \mathrm{X} 7$ receptor (P2X7R) subtype, coupled to a pore the opening of which allows entry of molecules up to $900 \mathrm{Da}$ inside cells, is assuming particular relevance in GBM and other tumors (Di Virgilio, 2020). P2X7R activation in glioma cells mediates multiple effects, ranging from cell death to survival and immune system modulation (D'Alimonte et al., 2015; Bergamin et al., 2019). Noteworthy, difference in the responses triggered by $\mathrm{P} 2 \mathrm{X} 7 \mathrm{R}$ highly depends on its expression levels (Andrejew et al., 2020). Accordingly, GSCs from primary GBMs show enhanced P2X7R expression (D'Alimonte et al., 2015) and in cells exposed to the P2X7R agonist, 2' (3')-O-(4benzoylbenzoyl)-ATP (BzATP), the expression of markers associated to epithelial-to-mesenchymal transition (EMT), a process contributing to GSC malignancy, was up-regulated as well as that of subunits of two main human P2X7R splice variants, P2X7A and P2X7B (Ziberi et al., 2019). This condition might favor A/B subunit assembly into a heterotrimeric P2X7R with major sensitivity toward agonists and cell energy support. All 
aforementioned findings suggest a crucial P2X7R role in GBM recurrence/invasiveness.

\section{ROLE OF ADENOSINE METABOLIZING ECTO-ENZYMES AND SIGNALS IN GLIOBLASTOMA AGGRESSIVENESS}

CD73 and Prostatic Acid phosphatase (PAP), which convert AMP to ADO, play a role in GBM progression. In particular, CD73 is expressed in GSCs from primary human tumors and glioma cell lines (D’Alimonte et al., 2015; Azambuja et al., 2019) and its overexpression is an important feature for glioma cell adhesion and tumor cell-extracellular matrix interactions (Cappellari et al., 2012). Accordingly, CD73 downregulation decreases GBM growth in vitro/in vivo models (Azambuja et al., 2019) and is coupled to a better outcome of GBM patients (Xu et al., 2013). Further data obtained in GSCs showed that AMP degradation by PAP is prevalent in hypoxic condition (Torres et al., 2019), increasing extracellular ADO levels (30-200 nM) up to 100 times (Uribe et al., 2017). As well, ADO levels measured in the extracellular fluid of glioma tissue from patients are elevated, being in the low micromolar range (Melani et al., 2003). Interestingly, increased ADO levels promoted tumorigenic GSC characteristics (Quezada et al., 2013; Garrido et al., 2014; Torres et al., 2016), while significantly increasing cell proliferation. In contrast, extracellular AMP, when present at high concentrations (1-3 mM), decreased proliferation of U138MG glioma cells lines (Bavaresco et al., 2008).

As for receptors involved in pro-/anti-tumorigenic $\mathrm{ADO}$ effects, all four receptor subtypes (namely, A1, A2A, A2B, and A3) are expressed in GSCs (D'Alimonte et al., 2015). The involvement of $\mathrm{ADO} /$ related analogues in the growth/ recurrence of gliomas gave rise to conflicting results about the role played by A1 and/or A2 receptors (see Gessi et al., 2011; Ceruti and Abbracchio, 2020). For example, some findings showed that $\mathrm{A} 1$ and $\mathrm{A} 2 \mathrm{~B}$ receptor stimulation had a prominent anti-proliferative/pro-apoptotic effect on GSCs via distinct regulation of the kinetics of ERK/AKT phosphorylation and the expression of hypoxia-inducible factors. Moreover, ADO receptor agonists sensitized GSCs to temozolomide (TMZ) toxicity and prolonged its effects (Daniele et al., 2014). In contrast, Yan et al. (2019) recently demonstrated that A2B receptor expression showed a 20 -fold increase in GBM cells implanted in mice brain and their blockade potently increased GBM cell death induced by TMZ by downregulating multidrug resistance transporter function. Likely, the differences above reported may depend on different experimental models used in the two studies. Noteworthy, both A2A and A2B receptors may be involved in the regulation of angiogenesis, indirectly supporting tumor growth with oxygen and nutrients (Gessi et al., 2011). In contrast, there is a more general agreement on the role of the low affinity $\mathrm{ADO} / \mathrm{A} 3$ receptors (A3R) on GBM growth/recurrence. Indeed, GSC migration and invasion was promoted by activation of these receptors especially in hypoxic condition (Torres et al., 2019). Accordingly, the blockade of
CD73 together with that of $\mathrm{ADO}$ receptors decreased the adhesion of cultured GBM cell lines (U138MG) to the extracellular matrix. A3R stimulation also promoted the expression of EMT markers in GSCs obtained from primary tumors and human U87MG cell line.

\section{ROLE OF INTRACELLULAR PURINE ALTERATIONS IN GLIOBLASTOMA MALIGNANCY}

In addition to alterations of membrane/extracellular purinome components, modifications of intracellular purine metabolism may also be relevant in GBM. For instance, the presence of high extracellular ADO levels usually activates its uptake by selective carriers, mainly equilibrative transporters (ENTs), whose driving force is the difference in nucleoside concentration across cell membrane. Thus, in rat $\mathrm{C} 6$ glioma cells at least, $\mathrm{ADO}$ uptake by these carriers, in particular ENT2 subtype, led to intracellular AMP accumulation due to activity of cytosolic ADO kinase functioning, however, only in oxygenation condition. AMP elevation, in turn, produced cell growth inhibition through pyrimidine starvation (Ohkubo et al., 2007). Other findings concern enzymes metabolizing other purines. Thus, in GBM ADF cell line, hyperactivity of the ubiquitous cytosolic enzyme 5'-nucleotidase II, converting inosines/guanosine monophosphate into the respective nucleosides, was accompanied by increased cell proliferation/resistance to gemcitabine and mitomycin C (Cividini et al., 2015). Finally, in GBM it was frequently found homozygous deletion of methylthioadenosine phosphorylase (MTAP), an enzyme working in the purine/methionine salvage pathway and metabolizing methylthioadenosine generated during polyamine biosynthesis to eventually produce adenine and methionine. MTAP alterations favor GSC formation with increased expression of the aggressiveness marker CD133 (Hansen et al., 2019), which is associated with poor clinical outcome of GBM patients (Zeppernick et al., 2008). Interestingly, $\mathrm{CD}_{133^{+}}$GSCs were killed by inhibiting de novo purine synthesis with L-alanosine, a potent low toxicity inhibitor of adenine biosynthesis, as reported in other tumors (Lubin and Lubin, 2009), coupled to ADO reuptake inhibition.

\section{DISCUSSION}

Being impossible to summarize the huge amount of data on GBM-purines relationship, we herein collected some impressive purinome dysfunctions in GBM. As for extracellular components, in our opinion two of them are crucial in supporting an oncogenic role of ATP/ADO in GBM. One is the activity of enzymes deputed to ATP or AMP metabolism, which is decreased or increased, respectively. This imbalance, coupled to enhanced purine loss from hypoxic/dying tumor cells, raises the extracellular ATP/ADO levels. The other one is a major expression of low affinity purine receptors, such as P2X7R or A3R, in GSCs and/or GBM cell lines, at least (Figure 1). The 
simultaneous presence of these alterations on the same tumor cell allows ATP/ADO to interact with receptors normally activated only by increased levels of the two purines, causing a more prolonged survival and/or aggressive behavior of the tumor itself.

While the role/modulation of CD39/CD73 activity in GBM still needs further elucidation in vivo experimental models/ patients and the extracellular purine level measurement is difficult, especially in vivo, given the rapidity by which these compounds are metabolized (nucleotides) or transported inside cells (nucleosides), at present search for A3R/P2X7R expression level in GBM surgical specimens seems more feasible, likely opening a new prognostic/ therapeutic scenario. Thus, in the presence of ascertained receptor overexpression, it could be useful to use selective A3R/P2X7R antagonists to curtail support to tumor growth/ malignancy. Noteworthy, the use of $\mathrm{P} 2 \mathrm{X} 7 \mathrm{R}$ antagonists might prove convenient also in inhibiting the onset of seizures occurring during GBM progression in patients, since ATP/ P2X7R interaction increases the release of ATP, triggering a vicious cycle, and also of glutamate (Strong et al., 2018), which may be toxic to the surrounding healthy brain neurons/tissue, also accounting for seizures (Corbetta et al., 2019). Finally, a high P2X7R expression is a good prognostic factor for glioma radiosensitivity and survival probability in humans (Gehring et al., 2015).

As well, alterations in GBM intracellular metabolism of purines/related substances can be important, in light of recent data showing that purine synthesis is fundamental for GSC maintenance (Wang et al., 2017). In our opinion, GBM MTAP deficiency is appealing. However, since tumor markers in addition to CD133 have been identified in GSCs (Lathia et al., 2015), studies on purine metabolism alterations should be carried out also in other GSC types and related intracranial xenograft animal models to assess the existence of MTAP loss or further/ different purine metabolism dysregulation in them. Afterward, it

\section{REFERENCES}

Andrejew, R., Oliveira-Giacomelli, Á., Ribeiro, D. E., Glaser, T., Arnaud-Sampaio, V. F., Lameu, C., et al. (2020). The P2X7 receptor: central hub of brain diseases. Front. Mol. Neurosci. 13, 124. doi:10.3389/fnmol.2020.00124

Azambuja, J. H., Gelsleichter, N. E., Beckenkamp, L. R., Iser, I. C., Fernandes, M. C., Figueiró, F., et al. (2019). CD73 downregulation decreases in vitro and in vivo glioblastoma growth. Mol. Neurobiol. 56 (5), 3260-3279. doi:10.1007/s12035018-1240-4

Bavaresco, L., Bernardi, A., Braganhol, E., Cappellari, A. R., Rockenbach, L., Farias, P. F., et al. (2008). The role of ecto-5'-nucleotidase/CD73 in glioma cell line proliferation. Mol. Cell. Biochem. 319 (1-2), 61-68. doi:10.1007/s11010-0089877-3

Bergamin, L. S., Capece, M., Salaro, E., Sarti, A. C., Falzoni, S., Pereira, M. S. L., et al. (2019). Role of the P2X7 receptor in in vitro and in vivo glioma tumor growth. Oncotarget 10 (47), 4840-4856. doi:10.18632/oncotarget.27106

Boison, D., and Yegutkin, G. G. (2019). Adenosine metabolism: emerging concepts for cancer therapy. Cancer Cell 36 (6), 582-596. doi:10.1016/j.ccell.2019.10.007

Braganhol, E., Wink, M. R., Lenz, G., and Battastini, A. M. O. (2020). Purinergic signaling in glioma progression. Adv. Exp. Med. Biol. 1202, 87-108. doi:10. 1007/978-3-030-30651-9

Burnstock, G. (2020). Introduction to purinergic signaling. Methods Mol. Biol 2041, 1-15. doi:10.1007/978-1-4939-9717-6 could be the case of evaluating the validity of modulating de novo purine synthesis/reuptake by ad hoc drugs.

In conclusion, the information collected here supports the idea that purinome alterations may have important consequences in events related to GBM aggressiveness/recurrence. Therefore, it is extremely important/needed to confirm whether these anomalies are present in vivo, so that they could be considered as new molecular markers of GBM and, hopefully, future targets for pharmacological/gene therapy.

\section{AUTHOR CONTRIBUTIONS}

RC conceived the work, drafted the manuscript, and composed the figure. MC and PG contributed in revising literature, writing and editing.

\section{FUNDING STATEMENT}

The funds from the University of Chieti-Pescara are the following: AT2019Ciccarelli assigned to RC.

\section{ACKNOWLEDGMENTS}

This opinion article, like many others in this Special Issue, is dedicated to Professor Geoffrey Burnstock, who was a pioneer in the research on purines, to which he devoted his entire life with great enthusiasm and scientific rigor. He has promoted interest in purine compound activity with an enormous amount of excellent scientific publications and with his constant participation in congresses dedicated to the role and activity of these substances. He will forever remain in the history of the purines and in the heart of those who had the good fortune to know him.

Burnstock, G. (2018). Purine and purinergic receptors. Brain Neurosci. Adv 2, 2398212818817494. doi:10.1177/2398212818817494

Campos-Contreras, A. D. R., Díaz-Muñoz, M., and Vázquez-Cuevas, F. G. (2020). Purinergic signaling in the hallmarks of cancer. Cells 9 (7), 1612. doi:10.3390/ cells9071612

Cappellari, A. R., Vasques, G. J., Bavaresco, L., Braganhol, E., and Battastini, A. M. (2012). Involvement of ecto-5'-nucleotidase/CD73 in U138MG glioma cell adhesion. Mol. Cell. Biochem. 359 (1-2), 315-322. doi:10.1007/s11010-0111025-9

Ceruti, S., and Abbracchio, M. P. (2020). Adenosine signaling in glioma cells. $A d v$. Exp. Med. Biol. 1202, 3-33. doi:10.1007/978-3-030-30651-9

Choi, J. S., and Berdis, A. J. (2012). Nucleoside transporters: biological insights and therapeutic applications. Future Med. Chem. 4 (11), 1461-1478. doi:10.4155/ fmc. 12.79

Cividini, F., Cros-Perrial, E., Pesi, R., Machon, C., Allegrini, S., Camici, M., et al. (2015). Cell proliferation and drug sensitivity of human glioblastoma cells are altered by the stable modulation of cytosolic $5^{\prime}$-nucleotidase II. Int. J. Biochem. Cell Biol. 65, 222-229. doi:10.1016/j.biocel.2015.06.011

Corbetta, C., Di Ianni, N., Bruzzone, M. G., Patanè, M., Pollo, B., Cantini, G., et al. (2019). Altered function of the glutamate-aspartate transporter GLAST.; a potential therapeutic target in glioblastoma. Int. J. Canc. 144 (10), 2539-2554. doi:10.1002/ijc.31985

D’Alimonte, I., Nargi, E., Zuccarini, M., Lanuti, P., Di Iorio, P., Giuliani, P., et al. (2015). Potentiation of temozolomide antitumor effect by purine 
receptor ligands able to restrain the in vitro growth of human glioblastoma stem cells. Purinergic Signal. 11 (3), 331-346. doi:10.1007/s11302-015-9454-7

Daniele, S., Zappelli, E., Natali, L., Martini, C., and Trincavelli, M. L. (2014) Modulation of $\mathrm{A} 1$ and $\mathrm{A} 2 \mathrm{~B}$ adenosine receptor activity: a new strategy to sensitise glioblastoma stem cells to chemotherapy. Cell Death Dis. 5 (11), e1539. doi:10.1038/cddis.2014.487

Di Virgilio, F. (2020) P2X7 is a cytotoxic receptor. ...maybe not: implications for cancer. Purinergic Signal. [Epub ahead of print]. doi:10.1007/s11302-02009735-w

Di Virgilio, F., Sarti, A. C., Falzoni, S., De Marchi, E., and Adinolfi, E. (2018). Extracellular ATP and P2 purinergic signalling in the tumour microenvironment. Nat. Rev. Cancer 18 (10), 601-618. doi:10.1038/s41568-018-0037-0

Fonta, C., Negyessy, L., Renaud, L., and Barone, P. (2004). Areal and subcellular localization of the ubiquitous alkaline phosphatase in the primate cerebral cortex: evidence for a role in neurotransmission. Cerebr. Cortex 14, 595-609. doi:10.1093/cercor/bhh021

Frenguelli, B. G., and Dale, N. (2020). Purines: from diagnostic biomarkers to therapeutic agents in brain injury. Neurosci Bull. [Epub ahead of print]. doi:10. 1007/s12264-020-00529-z

Garrido, W., Rocha, J. D., Jaramillo, C., Fernandez, K., Oyarzun, C., San Martin, R., et al. (2014). Chemoresistance in high-grade gliomas: relevance of adenosine signalling in stem-like cells of glioblastoma multiforme. Curr. Drug Targets 15 (10), 931-942. doi:10.2174/1389450115666140826122315

Gehring, M. P., Kipper, F., Nicoletti, N. F., Sperotto, N. D., Zanin, R., Tamajusuku, A. S., et al. (2015). P2X7 receptor as predictor gene for glioma radiosensitivity and median survival. Int. J. Biochem. Cell Biol. 68, 92-100. doi:10.1016/j.biocel. 2015.09.001

Gessi, S., Merighi, S., Sacchetto, V., Simioni, C., and Borea, P. A. (2011). Adenosine receptors and cancer. Biochim. Biophys. Acta 1808 (5), 1400-1412. doi:10.1016/ j.bbamem.2010.09.020

Giuliani, P., Zuccarini, M., Carluccio, M., Ziberi, S., Di Iorio, P., Caciagli, F., et al. (2018). A new investigational perspective for purines against glioblastoma invasiveness. Curr. Drug Targets 19 (16), 1871-1881. doi:10.2174/ 1389450119666180226123819

Hansen, L. J., Sun, R., Yang, R., Singh, S. X., Chen, L. H., Pirozzi, C. J., et al. (2019). MTAP loss promotes stemness in glioblastoma and confers unique susceptibility to purine starvation. Cancer Res. 79 (13), 3383-3394. doi:10. 1158/0008-5472.CAN-18-1010

Kakiuchi, M., Hirata, Y., Robson, S. C., and Fujisaki, J. (2020). Paradoxical regulation of allogeneic bone marrow engraftment and immune privilege by mesenchymal cells and adenosine. Biol. Blood Marrow Transplant [Epub ahead of print]. doi:10.1016/j.bbmt.2020.09.017

Lathia, J. D., Mack, S. C., Mulkearns-Hubert, E. E., Valentim, C. L., and Rich, J. N. (2015). Cancer stem cells in glioblastoma. Genes Dev. 29, 1203-1217. doi:10. 1101/gad.261982.115

Lubin, M., and Lubin, A. (2009). Selective killing of tumors deficient in methylthioadenosine phosphorylase: a novel strategy. PLoS One 4 (5), e5735. doi:10.1371/journal.pone.0005735

Melani, A., De Micheli, E., Pinna, G., Alfieri, A., Corte, L. D., and Pedata, F. (2003). Adenosine extracellular levels in human brain gliomas: an intraoperative microdialysis study. Neurosc. Lett. 346, 93-96. doi:10.1016/ S0304-3940(03) 00596-2

Morrone, F. B., Horn, A. P., Stella, J., Spiller, F., Sarkis, J. J., Salbego, C. G., et al. (2005). Increased resistance of glioma cell lines to extracellular ATP cytotoxicity. J. Neuro. Oncol. 71 (2), 135-1340. doi:10.1007/s11060-004-1383-1

Morrone, F. B., Oliveira, D. L., Gamermann, P., Stella, J., Wofchuk, S., Wink, M. R., et al. (2006). In vivo glioblastoma growth is reduced by apyrase activity in a rat glioma model. BMC Cancer 6, 226. doi:10.1186/1471-2407-6-226

Ohkubo, S., Nagata, K., and Nakahata, N. (2007). Adenosine uptake-dependent C6 cell growth inhibition. Eur. J. Pharmacol. 577, 35-43. doi:10.1016/j.ejphar.2007. 08.025

Quezada, C., Garrido, W., Oyarzún, C., Fernández, K., Segura, R., Melo, R., et al. (2013). 5'-Ectonucleotidases mediates multiple-drug resistance in glioblastoma multiforme cells. J. Cell. Physiol. 228 (3), 602-608. doi:10.1002/jcp.24168
Strong, A. D., Indart, M. C., Hill, N. R., and Daniels, R. L. (2018). GL261 glioma tumor cells respond to ATP with an intracellular calcium rise and glutamate release. Mol. Cell. Biochem. 446 (1-2), 53-62. doi:10.1007/s11010-018-3272-5

Taga, T., and Tabu, K. (2020). Glioma progression and recurrence involving maintenance and expansion strategies of glioma stem cells by organizing selfadvantageous niche microenvironments. Inflamm. Regen. 40, 33. doi:10.1186/ s41232-020-00142-7

Torres, A., Vargas, Y., Uribe, D., Jaramillo, C., Gleisner, A., Salazar-Onfray, F., et al. (2016). Adenosine A3 receptor elicits chemoresistance mediated by multiple resistance-associated protein-1 in human glioblastoma stem-like cells. Oncotarget 7 (41), 67373-67386. doi:10.18632/oncotarget.12033

Torres, Á., Erices, J. I., Sanchez, F., Ehrenfeld, P., Turchi, L., Virolle, T., et al. (2019). Extracellular adenosine promotes cell migration/invasion of Glioblastoma Stem-like Cells through $\mathrm{A}_{3}$ Adenosine Receptor activation under hypoxia. Cancer Lett. 446, 112-122. doi:10.1016/j.canlet.2019.01.004

Uribe, D., Torres, Á., Rocha, J. D., Niechi, I., Oyarzún, C., Sobrevia, L., et al. (2017). Multidrug resistance in glioblastoma stem-like cells: role of the hypoxic microenvironment and adenosine signaling. Mol. Aspect. Med. 55, 140-151. doi:10.1016/j.mam.2017.01.009

Wang, X., Yang, K., Xie, Q., Wu, Q., Mack, S. C., Shi, Y., et al. (2017). Purine synthesis promotes maintenance of brain tumor initiating cells in glioma. Nat. Neurosci. 20, 661-673. doi:10.1038/nn.4537

Wink, M. R., Lenz, G., Braganhol, E., Tamajusuku, A. S., Schwartsmann, G., Sarkis, J. J., et al. (2003). Altered extracellular ATP, ADP and AMP catabolism in glioma cell lines. Cancer Lett. 198 (2), 211-218.

Xu, S., Shao, Q. Q., Sun, J. T., Yang, N., Xie, Q., Wang, D. H., et al. (2013). Synergy between the ectoenzymes CD39 and CD73 contributes to adenosinergic immunosuppression in human malignant gliomas. Neuro Oncol. 15 (9), 1160-1172. doi:10.1093/neuonc/not067

Yan, A., Joachims, M. L., Thompson, L. F., Miller, A. D., Canoll, P. D., and Bynoe, M. S. (2019). CD73 promotes glioblastoma pathogenesis and enhances its chemoresistance via $\mathrm{A}_{2 \mathrm{~B}}$ adenosine receptor signaling. J. Neurosci. 39 (22), 4387-4402. doi:10.1523/JNEUROSCI.1118-18.2019

Yegutkin, G. G. (2014). Enzymes involved in metabolism of extracellular nucleotides and nucleosides: functional implications and measurement of activities. Crit. Rev. Biochem. Mol. Biol. 49 (6), 473-497. doi:10.3109/ 10409238.2014.953627

Zeppernick, F., Ahmadi, R., Campos, B., Dictus, C., Helmke, B. M., Becker, N., et al. (2008). Stem cell marker CD133 affects clinical outcome in glioma patients. Clin. Cancer Res. 14, 123-129. doi:10.1158/1078-0432.CCR-07-0932

Zhou, W., Yao, Y., Scott, A. J., Wilder-Romans, K., Dresser, J. J., Werner, C. K., et al. (2020). Purine metabolism regulates DNA repair and therapy resistance in glioblastoma. Nat. Commun. 11 (1), 3811. doi:10.1038/s41467-020-17512-x

Ziberi, S., Zuccarini, M., Carluccio, M., Giuliani, P., Ricci-Vitiani, L., Pallini, R., et al. (2019). Upregulation of epithelial-to-mesenchymal transition markers and $\mathrm{P} 2 \mathrm{X} 7$ receptors is associated to increased invasiveness caused by $\mathrm{P} 2 \mathrm{X} 7$ receptor stimulation in human glioblastoma stem cells. Cells 9 (1), 85. doi:10.3390/ cells 9010085

Zimmermann, H., Zebisch, M., and Sträter, N. (2012). Cellular function and molecular structure of ecto-nucleotidases. Purinergic Signal 8 (3), 437-502. doi:10.1007/s11302-012-9309-4

Disclaimer: This is an opinion article based only on literature review.

Conflict of Interest: The authors declare that the research was conducted in the absence of any commercial or financial relationships that could be construed as a potential conflict of interest.

Copyright ( 2021 Giuliani, Carluccio and Ciccarelli. This is an open-access article distributed under the terms of the Creative Commons Attribution License (CC BY). The use, distribution or reproduction in other forums is permitted, provided the original author(s) and the copyright owner(s) are credited and that the original publication in this journal is cited, in accordance with accepted academic practice. No use, distribution or reproduction is permitted which does not comply with these terms. 


\section{GLOSSARY}

ADO adenosine

AK adenosine kinase

A3R, A3 receptor

ATP, adenosine tri

ADP, adenosine di

AMP, adenosine mono-phosphate

BzATP, 2'(3')-O-(4-benzoylbenzoyl)-ATP

EMT epithelial-to-mesenchymal transition

E-NPPases ectonucleoside pyrophosphatase/phosphodiesterases

ecto-5' - NT/CD73 ecto-5' -nucleotidase/CD73
E-NTPDases ectonucleoside triphosphate diphosphohydrolase

ENTs equilibrative transporters

GBM glioblastoma multiforme

GSCs glioblastoma stem cells

MTA methylthioadenosine

MTAP methylthioadenosine phosphorylase

NTAP tissue-nonspecific alkaline phosphatase

NTs nucleotidases

PAP prostatic acid phosphatase

P2X7R, P2X7 receptor

SAH S-adenosyl hydroxylase 